\title{
The Relationships between Morphological Characteristics and Foraging Behavior in Four Selected Species of Shorebirds and Water Birds Utilizing Tropical Mudflats
}

\author{
Nor Atiqah Norazlimi and Rosli Ramli \\ Institute of Biological Sciences, Faculty of Science, University of Malaya, 50603 Kuala Lumpur, Malaysia \\ Correspondence should be addressed to Nor Atiqah Norazlimi; noratiqahnorazlimi@gmail.com
}

Received 26 May 2015; Revised 28 July 2015; Accepted 29 July 2015

Academic Editor: Antonio J. Piantino Ferreira

Copyright (C) 2015 N. A. Norazlimi and R. Ramli. This is an open access article distributed under the Creative Commons Attribution License, which permits unrestricted use, distribution, and reproduction in any medium, provided the original work is properly cited.

A study was conducted to investigate the relationship between the physical morphology of shorebirds and water birds (i.e., Lesser adjutant (Leptoptilos javanicus), Common redshank (Tringa totanus), Whimbrel (Numenius phaeopus), and Little heron (Butorides striata)) and their foraging behavior in the mudflats area of Selangor, Peninsular Malaysia, from August 2013 to July 2014 by using direct observation techniques (using binoculars and a video recorder). The actively foraging bird species were watched, and their foraging activities were recorded for at least 30 seconds for up to a maximum of five minutes. A Spearman Rank Correlation highlighted a significant relationship between bill size and foraging time $(R=0.443, p<0.05)$, bill size and prey size $(R=-0.052$, $p<0.05)$, bill size and probing depth $(R=0.42, p=0.003)$, and leg length and water/mud depth $(R=0.706, p<0.005)$. A Kruskal-Wallis Analysis showed a significant difference between average estimates of real probing depth of the birds (mm) and species $(H=15.96, p=0.0012)$. Three foraging techniques were recorded: pause-travel, visual-feeding, and tactile-hunting. Thus, morphological characteristics of bird do influence their foraging behavior and strategies used when foraging.

\section{Introduction}

Shorebirds are a highly mobile group of animals and have sophisticated site-sampling processes that operate on larger spatial scales than most other animals [1]. Shorebirds generally forage during low tide and can be observed on beaches, intertidal mudflats, freshwater and brackish wetlands, farmland, and salt marshes [2]. Meanwhile, water birds refer to the bird species that entirely depend on wetlands for a variety of activities such as foraging, nesting, loafing, and moulting [3]. Both shorebirds and water birds are the important components of estuarine mudflats.

Estuarine mudflats are very important for many shorebird populations during winter and migration, many species of which feed almost exclusively on intertidal benthic invertebrates at low tide [4]. Besides that, mudflats in estuaries are also vital feeding habitats for resident bird populations [5]. In tropical regions, the biodiversity of benthic macrofauna on intertidal mudflats is much higher; macrofauna are produced ten times faster here than in temperate intertidal habitats [6,
7]. During migratory seasons, foraging is the most important activity for shorebirds utilizing the mudflats area, as it allows them to survive and ensures their safe arrival at the breeding ground. The foraging ecology is often characterized by food selection, habitat preference, and prey capturing tactics or behavior employed by avian species in a particular habitat $[8,9]$.

The morphology of a bird is considered an important factor in restricting the ranges of foraging maneuvers it can perform $[10,11]$. Bill length and shape have important implications on foraging behavior [12-15], microhabitat selection [16], and choice of diet [15, 17-19]. Longer bills are associated with probing depth and plunging or sweeping bill movements in the water, while shorter bills are associated with routing and pecking at the substrate surface [14]. Furthermore, the foraging depths are also correlated with culmen and tarsus lengths [20]. The bill's shape (either straight or curved) influences the foraging techniques used by Calidris mauri (Western sandpipers) [15]. Pecking or feeding on epifaunal invertebrates is associated with a straight bill, while probing 
or feeding on infaunal prey is facilitated by bill curvature. In terms of foraging strategies, the functional requirement of a tactile foraging strategy is a high penetration capacity, which is then influenced by the morphological characters of a bird's bill [13]. The general morphological requirements necessitate that the bill be long and narrow but not very slender, and the penetrating portion should be flattened either vertically or horizontally. Time spent feeding also varies with respect to the size of the bird [21]. Larger birds spent less time foraging than smaller birds by eating larger and more profitable prey.

The majority of the studies on the foraging behavior of shorebirds and water birds were conducted in temperate climate areas. The feeding ecology of shorebird and water birds species in tropical countries, especially Malaysia, is poorly understood. A previous study [28] focused on the correlation between bird density and prey density, whereas one study [29] focused on birds' habitat utilization. To date, no detailed information has been obtained on the correlation between the morphological characteristics of birds and their feeding ecology in Malaysia. Therefore, this study aims to determine the significant relationships between morphological characters and foraging behavior adapted by shorebird and water birds species utilizing the mudflats area of Jeram Beach and Remis Beach, Selangor, Peninsular Malaysia.

\section{Materials and Methods}

2.1. Study Area. The Jeram and Remis Beaches are located on the Selangor Coast on the West Coast of Peninsular Malaysia $\left(3^{\circ} 13^{\prime} 27^{\prime \prime} \mathrm{N}, 101^{\circ} 18^{\prime} 13^{\prime \prime} \mathrm{E}\right)$ (Figure 1) where semidiurnal tides prevail. In Jeram Beach, the flat was fringed by a mangrove stand of stunted Avicennia alba Blume and few scattered Sonneratia spp. [30]. The distance between Jeram Beach and Remis Beach is approximately $2 \mathrm{~km}$. The selected study areas comprise approximately 55 ha of intertidal mudflats, that is, 27 ha on Jeram Beach and 28 ha on Remis Beach. The selection of these sites was based on past shorebird counts reported by Wetland Internationals from 1999 to 2004 [31], which shows that these areas were previously known to be important stopover sites for shorebirds.

2.2. Foraging Behavior. The foraging behavior of birds was studied from August 2013 to July 2014 using direct observation techniques. Selected focal birds were observed using binoculars (12 $\times 42$ magnifications), stopwatches, and video recorders. The selected focal bird must be actively foraging (each individual was observed until they were done foraging, i.e., starting from the time the bird began actively searching for prey until the prey was completely swallowed); if the bird left within 30 seconds, it was eliminated from the study [32]. The focal observations were recorded for at least 30 seconds for up to a maximum of five minutes. The data recorded from the different sites and months were pooled to increase replications [33] so that the data was strong enough to be analyzed. The focal observations were done only during low tide period (i.e., during ebbing tide, low tide peak, and rising tide) so that birds of all sizes (either with longer or shorter legs) can use the mudflats area for foraging at the same time.

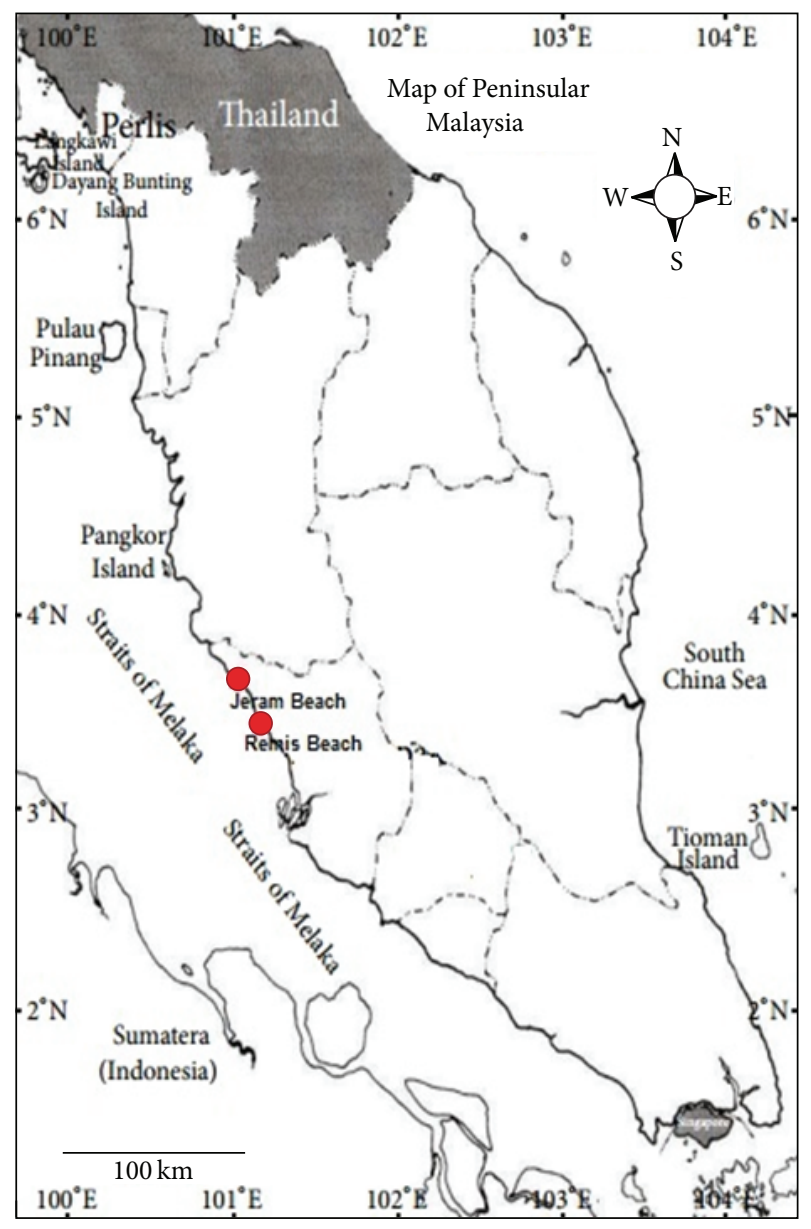

Figure 1: The Coastal Mudflats of Jeram and Remis Beaches, Selangor, West Coast of Peninsular Malaysia.

The observations were conducted during four-interval period (i.e. 0800-1000 hours, 1000-1200 hours, 1400-1600 hours, and 1600-1800 hours). Since the sites were situated nearest to each other (i.e., $2 \mathrm{~km}$ apart), the differentiation of habitat characteristics was not obvious. First, individual focal birds were selected from a flock. Once a bird was chosen, the next bird selected for observation must be located at least 10 meters away from the previously observed bird. This was done to avoid multiple observations of the same individual. Four species of shorebirds and water birds (Lesser adjutant (Leptoptilos javanicus), Common redshank (Tringa totanus), Little heron (Butorides striata), and Whimbrel (Numenius phaeopus)) were chosen for this study due to their size differences and foraging techniques and because they are easily distinguished from one another. The data, such as estimated probing depth, time spent foraging (total searching and handling time), foraging techniques, prey type, estimated prey size, and estimated water or mud depth in which they forage, were gathered. Foraging techniques used by bird were divided into three categories: (1) tactile-hunting species techniques, where birds forage as they walk, probing continuously with the bill into the substrate $[34,35]$; (2) visual-feeding techniques, where they forage in a continuous 
TABLE 1: Measurements of bill size and tarsus length based on previous studies.

\begin{tabular}{|c|c|c|c|c|c|}
\hline Species & Literature & $\begin{array}{l}\text { Bill length } \\
(\mathrm{mm})\end{array}$ & $\begin{array}{l}\text { Average bill length } \\
(\mathrm{mm})\end{array}$ & $\begin{array}{c}\text { Tarsus length } \\
(\mathrm{mm})\end{array}$ & $\begin{array}{l}\text { Average tarsus length } \\
\qquad(\mathrm{mm})\end{array}$ \\
\hline Lesser adjutant & [22] & 266.7 & 266.7 & 228.6 & 228.6 \\
\hline Common redshank & {$[23,24]$} & $\begin{array}{l}43.7 \\
41.8\end{array}$ & 42.8 & $\begin{array}{l}51.6 \\
47.6\end{array}$ & 49.6 \\
\hline Little heron & {$[25]$} & 75.0 & 75.0 & 49.0 & 49.0 \\
\hline Whimbrel & {$[26,27]$} & $\begin{array}{l}87.2 \\
82.0\end{array}$ & 84.6 & $\begin{array}{l}55.9 \\
63.8\end{array}$ & 59.9 \\
\hline
\end{tabular}

TABLE 2: Summary of frequency of individual of shorebird and water bird species observed $(n)$ from August 2013 until July 2014.

\begin{tabular}{|c|c|c|c|c|}
\hline \multirow[b]{2}{*}{ Months } & \multicolumn{4}{|c|}{ Species $(n)$} \\
\hline & $\begin{array}{c}\text { Lesser } \\
\text { adjutant }\end{array}$ & $\begin{array}{l}\text { Common } \\
\text { redshank }\end{array}$ & $\begin{array}{l}\text { Little } \\
\text { heron }\end{array}$ & Whimbrel \\
\hline August & 6 & 4 & 8 & 4 \\
\hline September & 10 & 20 & 2 & 3 \\
\hline October & 11 & 42 & 2 & 2 \\
\hline November & 3 & 23 & 4 & 2 \\
\hline December & 7 & 33 & 7 & 4 \\
\hline January & 9 & 19 & 5 & 4 \\
\hline February & 5 & 17 & 3 & 4 \\
\hline March & 5 & 17 & 5 & 3 \\
\hline April & 5 & 11 & 5 & 3 \\
\hline May & 6 & 10 & 5 & 3 \\
\hline June & 4 & 5 & 4 & 3 \\
\hline \multirow[t]{2}{*}{ July } & 4 & 4 & 3 & 3 \\
\hline & 75 & 205 & 53 & 38 \\
\hline
\end{tabular}

fashion, pecking at items seen on the substrate surface [36] and; (3) pause-travel species techniques, where they mainly forage by scanning the area in front of them and pecking at the substrate surface when prey is detected in a stop-runstop fashion [37, 38]. Five prey items were observed to be the main diet choice for shorebirds: fish, bivalves, worms, crabs, and unknown (aquatic insects or invertebrate fauna). Preys were classified as "unknown" when they could not be clearly seen. The size and number of prey items taken by these birds were estimated and recorded. Bill length was used to estimate probing depth and prey size, while leg length was used to estimate water or mud depth based on the maximum percentage (\%) of the leg that was immersed in the water or mud. The length of the leg of selected birds was estimated by doubling the length of the tarsus. Similarly, the probing depth was estimated based on the maximum percentage of bill length inserted into the mud or water. Meanwhile, prey size was estimated based on the percentage of the prey items inserted into the bill. Table 1 summarizes the measurement of the bill size and tarsus length of the bird species based on previous studies. Below are the formulae used to illustrate the estimation of probing depth and water or mud depths:

Water/mud depth (mm)

$=\underline{\text { Percentage of estimated leg immersed in the water or mud }}$

$\quad \times$ leg length,
Probing depth $(\mathrm{mm})$
$=\frac{\text { Percentage of estimated bill inserted into the mud or water }}{100 \%}$
$\quad \times$ bill size,

Prey size $(\mathrm{mm})$

$=\frac{\text { Percentage of estimated prey in bill }}{100 \%} \times$ bill size.

2.3. Data Analysis. Statistical software [39] was used to analyze all data. In preparation for statistical testing, all data sets were tested with Shapiro Wilk's $W$ test and Anderson's Darling test for normality. In all cases, $\alpha=0.05$ was used. A total of 205 focal observations were recorded for Common redshank, 75 observations for Lesser adjutant, 53 observations for Little heron, and 38 observations for Whimbrel (Table 2). Due to differences in number of focal observations recorded, all data taken were divided into 12 months (i.e., from August 2013 until July 2014) to obtain the average or mean of each data. A Spearman Rank Correlation Analysis was used to determine the correlation between the bill size of the bird and the time spent foraging [40]; the bill size of the bird and the estimated prey size; the bill size of the bird and probing depth; and the leg length of the bird and water or mud depth. The nonparametric Kruskal-Wallis Test was used to study the relationships between bird species and probing depth $(\mathrm{mm})$. Moreover, a one-way ANOVA was used to determine the differences in time spent foraging and different foraging techniques. All the requisites of data reliability have been followed [41]. The statistical test used was based on [42].

\section{Results}

Our results show that time of the day and tidal conditions do not influence the use of habitat for foraging by shorebird 
TABLE 3: Summary of bill size, average estimated probing depth, length of leg, average estimated water/mud depth per year, and average time spent foraging by shorebirds and water birds.

\begin{tabular}{lccccc}
\hline Species & $\begin{array}{c}\text { Bill size } \\
(\mathrm{mm})\end{array}$ & $\begin{array}{c}\text { Estimated probing/ } \\
\text { foraging depth }(\mathrm{mm})\end{array}$ & $\begin{array}{c}\text { Length of the } \\
\text { leg }(\mathrm{mm})\end{array}$ & $\begin{array}{c}\text { Estimated water/ } \\
\text { mud depth }(\mathrm{mm})\end{array}$ & $\begin{array}{c}\text { Time spent } \\
\text { foraging }(\mathrm{s})\end{array}$ \\
\hline Little heron & 75 & 24 & 98 & 27 & 1,130 \\
Lesser adjutant & 266.7 & 82 & 457.2 & 134 & 3,186 \\
Whimbrel & 84.6 & 41 & 119.8 & 22 & 38 \\
Common redshank & 42.8 & 33 & 99.2 & 2,085 \\
\hline
\end{tabular}

TABLE 4: Diet choice and abundance chosen by shorebird and water bird species.

\begin{tabular}{lcc}
\hline Species & Prey type & Number of prey counted \\
\hline \multirow{4}{*}{ Little heron } & Fish & 35 \\
& Bivalve & 0 \\
& Worm & 0 \\
& Crab & 0 \\
& Unknown & 17 \\
\hline \multirow{4}{*}{ Lesser adjutant } & Fish & 51 \\
& Bivalve & 7 \\
& Worm & 2 \\
& Crab & 2 \\
& Unknown & 15 \\
\hline \multirow{4}{*}{ Whimbrel } & Fish & 8 \\
& Bivalve & 17 \\
& Worm & 1 \\
& Crab & 7 \\
& Unknown & 0 \\
\hline \multirow{4}{*}{ Common redshank } & Fish & 13 \\
& Bivalve & 102 \\
& Worm & 25 \\
& Crab & 34 \\
& Unknown & 35 \\
\hline
\end{tabular}

and water bird species utilizing the areas of the study. Oneway ANOVA analysis was conducted between four intervals periods (i.e., 0800-1000, 1000-1200, 1400-1600, and 16001800 hours) and the results show no significant difference in the time of birds species that used the habitat for foraging during the intervals $(F=1.86, p=0.190)$. Similarly, no significant differences were found during different low tide period (i.e., ebbing tide, low tide peak, and rising tide) $(F=$ $1.62, p=0.251$ ). Furthermore, by using Friedman's TwoWay Analysis of Variance by Ranks Test, the results show no significant difference for searching time of preys between species $(F=4.92, p=0.178)$. On the contrary, the handling times were significantly different between the bird species $(F=19.49, p<0.05)$. Further analysis by using Pairwise Comparisons Test highlighted that the differences occurred between Lesser adjutant and Whimbrel $(z=-1.7, p=0.019)$ and also between Common redshank and Little heron $(z=$ $1.6, p=0.034)$. The differences in handling time were found to influence the total time spent for foraging between the birds species $(F=13.3, p=0.004)$. Pairwise Comparisons
Test proved that the significant difference occurred between Little heron and Lesser adjutant $(z=-1.667, p=0.009)$ and between Lesser adjutant and Common redshank $(z=$ $1.583, p=0.016)$. In this study, we found that the time spent foraging, prey size, and probing depth differed with respect to the bill size of the shorebird and water bird species. Moreover, the water or the mud depth where the bird stood while foraging was also influenced by the length of the leg of the bird species. A Spearman Rank Correlation shows a significant relationship between bill size and time spent foraging $(R=0.443, p<0.05)$; the bill sizes and the estimated prey size obtained while foraging $(R=-0.052, p<0.05)$; the bill sizes and the probing depth applied while foraging $(R=0.42, p=0.003)$; and the length of the leg and the water or mud depth in the feeding area $(R=0.706, p<0.005)$ (Table 3).

We also found that probing depth varied between species. A Kruskal-Wallis Analysis shows a significant difference between average estimates of real probing depth $(\mathrm{mm})$ and species $(H=15.96, p=0.0012)$. Bonferroni's post hoc test, with a correction of $\alpha=0.05$, was calculated, and the results showed that the significant difference exists only between the Little heron and the Lesser adjutant $(z=3.97, p=0.001)$.

In terms of diet choice, five prey items were observed to be the main diet choice for shorebirds and water birds: fish, bivalves, worms, crabs, and unknown (Table 4). Based on observations, the bivalve was the most preferred option among bird species, which accounted for $34 \%$ of the diet, followed by fish, $29 \%$, unknown, $18 \%$, crab, $12 \%$, and worm, $7 \%$.

Table 5 shows foraging techniques practiced by shorebird and water bird species. Little herons only practiced pause-travel techniques, while Lesser adjutants and Common redshanks used all techniques while foraging. However, the most preferred feeding technique used by Lesser adjutants and Common redshanks was the tactile-hunting feeding technique. In contrast, the Whimbrel engaged in both tactilehunting and visual-feeding techniques, but not pause-travel techniques. No significant differences were found between time spent foraging and different feeding techniques $(F=$ $0.26, p=0.778)$.

\section{Discussion}

We found that time of the day and tidal conditions do not influence the foraging behavior of shorebird and water bird species utilizing the areas of the study. Similar results were 
TABLE 5: Sample size $(n)$, mean and standard error of time spent foraging, and foraging techniques used by species.

\begin{tabular}{lcccc}
\hline \multirow{2}{*}{ Species } & Foraging techniques & \multicolumn{3}{c}{ Time spent foraging (s) } \\
& & $n$ & Mean & Standard error \\
\hline \multirow{2}{*}{ Little } & Pause-travel & 53 & 68.62 & 5.69 \\
heron & Tactile-hunting & 0 & 0.00 & 0.00 \\
& Visual-feeding & 0 & 0.00 & 0.00 \\
\hline \multirow{2}{*}{ Lesser } & Pause-travel & 17 & 134.65 & 18.50 \\
adjutant & Tactile-hunting & 56 & 77.34 & 8.13 \\
& Visual-feeding & 2 & 24.00 & 4.00 \\
\hline \multirow{3}{*}{ Whimbrel } & Pause-travel & 0 & 0.00 & 0.00 \\
& Tactile-hunting & 33 & 36.70 & 2.03 \\
& Visual-feeding & 5 & 120.00 & 0.00 \\
\hline \multirow{2}{*}{ Common } & Pause-travel & 2 & 50.00 & 0.00 \\
redshank & Tactile-hunting & 171 & 46.09 & 2.58 \\
& Visual-feeding & 32 & 39.53 & 2.54 \\
\hline
\end{tabular}

found by [43] through her study of tidal flats, which showed that time of day did not significantly affect variability in shorebird abundance and use of habitat. Previous study by [44] found that the abundance of the birds reached peak between 1.5 and 2.5 hours after low tide which suggests that the availability of food is the greatest during this period. However, surprisingly, our results were in contrast with this study. Any subtle differences in the bird's morphological traits, such as the length of the wing, tarsus, or toes, could result in different foraging maneuvers [45]. This study revealed that the differences in bill size and leg length of the shorebird and water bird species influence the time spent foraging, the size of the prey, the probing depth, and the preference of habitat while foraging. The longer the bill, the more time spent foraging, the larger the prey, and the deeper the area they preferred to forage. Based on our observations, Lesser adjutants and Little herons had longer bills and their diets mainly comprised of larger prey items such as fish. Larger prey required longer swallowing and digesting times, allowing birds to spend more time foraging. Increasing the time spent handling the prey resulted in an increase in the time spent foraging. On the contrary, birds with shorter bills (the Common redshank) were observed to feed on bivalves more frequently. Smaller prey reduced handling time and, thus, reduced time spent foraging. Similar results have been reported [46], which show that birds with longer bills generally feed on larger prey than birds with shorter bills. Probing depth was hypothesized to increase as the length of the bird's bill increased. Birds with longer bills (Lesser adjutants) were observed to probe in deeper mud and higher water as compared to other bird species. A study of the differences in bill sizes of male and female Western Sandpipers (Calidris mauri) [47] found that females, who have longer bills, foraged in sites with a higher water content than males did, where the probing technique may be more effective [16]. Although birds with longer bills probed deeper than shorter billed birds, the percentage of which the bill inserted into the mud or water while foraging was differed.
Shorter billed birds tended to insert the majority or all of their bills into the mud or water while foraging. Usually, the Common redshank inserted the majority of its bill into the mud while foraging, whereas the Lesser adjutant, Whimbrel, and Little heron only inserted their bills halfway or less while foraging. Deeper probing resulted in a more profitable prey item. The size of the prey increased with respect to burrowing depth. A previous study [48] found that a larger worm species (Nereis diversicolor), which is longer than $10 \mathrm{~cm}$, was usually found at a depth of 10 to $14 \mathrm{~cm}$.

The longer the leg, the deeper the mud or water depth in which the birds stood while foraging. This study revealed that the Lesser adjutant tended to forage in the deeper mud and areas close to the water's edge. Meanwhile, the Common redshank was commonly found utilizing the area closest to the beach, which was shallower and drier compared to the area closest to the water's edge. Similar results [49] show that shorebirds with shorter legs and tarsi (i.e., Calidris minutilla (Least Sandpiper), Calidris mauri (Western Sandpiper), Limnodromus spp. (Dowitcher), and Calidris alpina (Dunlin)) were constrained to use mudflats or shallow water zones along the wetland's edge. Leg length was positively correlated with water depth in which shorebirds foraged [20]. Other data [50] also revealed an increase in the range of depths used by larger shorebird species, which wade in deeper habitats. Foraging close to the water's edge might be advantageous because of increased penetrability and prey activity [51]. Therefore, drier substrates and more structurally complex microhabitats may be favored by birds with shorter bills $[52,53]$.

Our results show a significant difference between average estimates of probing depth and species. The differences in probing depth exist only between Little herons and Lesser adjutants. This may be due to differences in their bill sizes. Lesser adjutants have longer bills than Little herons. Birds with longer bills will benefit by probing deeper into the mud. The differences in habitat use exist in sandpipers due to variations in bill length [54]; that is, longer billed individuals foraged in muddier habitats than did shorter billed individuals.

The foraging techniques engaged in while foraging also differed between species. Tactile hunting was the most dominant technique used by the Lesser adjutant, Whimbrel, and Common redshank, whereas the pause-travel technique was the only technique used by the Little heron. The different types of feeding techniques are likely to influence the vigilance patterns of shorebird species. Pause-travel species can be more vigilant with their heads up, scanning the environment; when they locate a prey item, they run to catch it [55]. We assume that tactile-hunting techniques increase the chances of successful foraging, since much of the bird's time is concentrated on searching for food, compared to pause-travel techniques, in which the bird spends much of its time being more vigilant than foraging. Moreover, shorter billed birds were restricted to a certain mud depth or water level compared to the longer billed bird. Therefore, tactilehunting techniques were observed to be the most profitable, since the bird using this technique will probe as deep as possible to obtain more profitable prey, which burrow deep 
into the mud. Our study suggests that time spent foraging did not differ between foraging techniques. However, different results have been shown [56], where Plovers, which exhibit visual foraging techniques, spend less time feeding than Sandpipers, which exhibit tactile or continuous hunting techniques. Furthermore, the pause-travel species was frequently observed foraging alone, whereas tactile and visual feeding species usually foraged in intraspecies or interspecies flocks. Foraging in groups is beneficial because it reduces the risk of predation and, thus, reduces the cost of vigilance [32]. For conclusion, the morphologies of birds play an important role in determining foraging behaviors. Species with different foraging strategies will acquire food resources from different habitats and may be able to avoid interspecies competition. Thus, sufficient energy and nutrients can be replenished to enhance the survival of bird species in the area.

\section{Conflict of Interests}

Both authors declare that there is no conflict of interests regarding the publication of this paper.

\section{Acknowledgments}

The authors thank the staff at the Institute of Biological Science for assistance in the field. This study was funded by a University of Malaya Research Grant (PG031-2013B) and a MyBrainSc Scholarship from the Ministry of Education, Malaysia.

\section{References}

[1] G. Quaintenne, J. A. Van Gils, P. Bocher, A. Dekinga, and T. Piersma, "Scaling up ideals to freedom: are densities of red knots across western Europe consistent with ideal free distribution?" Proceedings of the Royal Society B: Biological Sciences, vol. 278, no. 1719, pp. 2728-2736, 2011.

[2] B. Lane, Shorebirds in Australia, Nelson Publishers, Melbourne, Australia, 1987.

[3] M. N. Rajpar and M. Zakaria, "Density and diversity of water birds and terrestrial birds at Paya Indah Wetland Reserve, Selangor Peninsular Malaysia," Journal of Biological Sciences, vol. 10, no. 7, pp. 658-666, 2010.

[4] P. L. A. Erftemeijer and R. Lewis, "Planting mangroves on intertidal mudflats: habitat restoration or habitat conversion?" in Proceedings of the 8th ECOTONE Seminar"Enhancing Coastal Ecosystem Restoration for the 21st Century", pp. 1-13, Ranong and Phuket, Thailand, 1999.

[5] J. D. Goss-Custard and N. Verboven, "Disturbance and feeding shorebirds on the Exe estuary," Wader Study Group Bulletin, vol. 68, pp. 59-66, 1993.

[6] D. Alongi, "The ecology of tropical soft-bottom benthic ecosystems," in Oceanography and Marine Biology: An Annual Review, vol. 28, pp. 381-496, 1990.

[7] A. D. Ansell, D. S. McLusky, A. Stirling, and A. Trevallion, "Production and energy flow in the macrobenthos of two sandy beaches in South West India," Proceedings of the Royal Society of Edinburgh. Section B: Biological Sciences, vol. 76, no. 04, pp. 269-296, 1978.
[8] E. Danchin, L. Giraldeau, and F. Cézilly, Behavioural Ecology, Oxford University Press, New York, NY, USA, 2008.

[9] A. B. H. Aboushiba, R. Ramli, and M. S. Azirun, "Foraging behaviour of five egret species in POME Pond Area at Carey Island, Peninsular Malaysia," The Journal of Plant \& Plant Sciences, vol. 23, no. 1, pp. 129-135, 2013.

[10] T. E. Martin and J. R. Karr, "Behavioral plasticity of foraging maneuvers of migratory warblers: multiple selection periods for niches?" Studies in Avian Biology, vol. 13, pp. 353-359, 1990.

[11] M. C. K. Soh, "Foraging behaviour of two tailorbirds in Singapore: habitat, morphological and temporal comparisons," The Raffles Bulletin of Zoology, vol. 49, no. 2, pp. 173-180, 2001.

[12] J. P. Pierre, "Effects of sexual dimorphism on feeding behaviour of the bar-tailed godwit (Limosa lapponica) at a Southern Hemisphere wintering site," New Zealand Natural Sciences, vol. 21, pp. 109-112, 1994.

[13] A. A. Gerritsen and G. F. C. Zweers, "Transitions from pecking to probing mechanisms in waders," Netherlands Journal of Zoology, vol. 47, no. 2, pp. 161-208, 1996.

[14] A. Barbosa and E. Moreno, "Evolution of foraging strategies in shorebirds: an ecomorphological approach," The Auk, vol. 116, no. 3, pp. 712-725, 1999.

[15] S. Nebel, D. L. Jackson, and R. W. Elner, "Functional association of bill morphology and foraging behaviour in calidrid sandpipers," Animal Biology, vol. 55, no. 3, pp. 235-243, 2005.

[16] Y. Van Heezik and A. Gerritsen, "Substrate preference and substrate related foraging behavior in three Calidris species," Netherlands Journal of Zoology, vol. 35, no. 4, pp. 671-692, 1984.

[17] B. Lauro and E. Nol, "Feeding behavior, prey selection and bill size of Pied and Sooty Oystercatchers in Australia," Wilson Bulletin, vol. 107, pp. 629-640, 1995.

[18] V. Mascitti and F. O. Kravetz, "Bill morphology of South American flamingos," The Condor, vol. 104, no. 1, pp. 73-83, 2002.

[19] D. Durant, H. Fritz, S. Blais, and P. Duncan, "The functional response in three species of herbivorous Anatidae: effects of sward height, body mass and bill size," Journal of Animal Ecology, vol. 72, no. 2, pp. 220-231, 2003.

[20] M. C. Baker, "Morphological correlates of habitat selection in a community of shorebirds (Charadriiformes)," Oikos, vol. 33, no. 1, pp. 121-126, 1979.

[21] Y. Ntimao-Baidu, T. Piersma, P. Wiersma, M. Poot, P. Battley, and C. Gordon, "Water depth selection, daily feeding routines and diets of waterbirds in coastal lagoons in Ghana," Ibis, vol. 140, no. 1, pp. 89-103, 1998.

[22] J. A. Murray, The Avifauna of the Island of Ceylon, Educational Society Press, Bombay, India, 1890.

[23] W. G. Hale, F. Scarton, and R. Valle, "The taxonomic status of Redshank Tringa totanus in Italy," Bulletin of British Ornithologist's Club, vol. 125, no. 4, pp. 261-275, 2005.

[24] P. S. Thompson, C. McCarty, and W. G. Hale, "Growth and development of redshank Tringa totanus chick on the Ribble Saltmarshes, N.W. England," Ringing \& Migration, vol. 11, no. 1, pp. 57-64, 1990.

[25] D. R. Wells, The Birds of the Thai-Malay Peninsula: NonPasserines, Academic Press, London, UK, 1999.

[26] A. Poole and F. Gill, The Birds of North America, The Birds of North America, Philadelphia, Pa, USA, 2000.

[27] V. V. Morozov, "Current status of the southern subspecies of the Whimbrel Numenius phaeopus albazxillaris (Lowe 1921) in 
Russia and Kazakstan," Wader Study Group Bulletin, vol. 92, pp. 30-37, 2000.

[28] R. M. Lomoljo, Diversity of migratory shorebirds and their habitat characteristics in Kuala Gula Bird Sanctuary, Perak, Malaysia [Dissertation for Doctoral Degree], Universiti Putra Malaysia, Seri Kembangan, Malaysia, 2011.

[29] K. M. Riak, An ecological assessment of wetland habitat utilized by migratory shorebirds at Kapar, Pantai Remis and Kuala Selangor Nature Park, Malaysia [Ph.D. thesis], Universiti Putra Malaysia, Seri Kembangan, Malaysia, 2004.

[30] G. Polgar, “The polychaete reefs of Jeram, Selangor," in Ecology of Klang Strait, Faculty of Science, University of Malaya, Kuala Lumpur, Malaysia, 2nd edition, 2012.

[31] Z. W. D. Li and R. Ounsted, The Status of Coastal Waterbirds and Wetlands in Southeast Asia: Results of Waterbirds Surveys in Malaysia (2004-2006) and Thailand and Myanmar (2006), Wetland International, Kuala Lumpur, Malaysia, 2007.

[32] J. Burger, S. A. Carlucci, C. W. Jeitner, and L. Niles, "Habitat choice, disturbance, and management of foraging shorebirds and gulls at a migratory stopover," Journal of Coastal Research, vol. 235, no. 5, pp. 1159-1166, 2007.

[33] L. Green, D. T. Blumstein, and P. Fong, "Macroalgal mats in a eutrophic estuary obscure visual foraging cues and increase variability in prey availability for some shorebirds," Estuaries and Coasts, vol. 38, no. 3, pp. 917-926, 2015.

[34] A. F. C. Gerritsen and J. G. Sevenster, "Foraging behavior and bill anatomy in sandpipers," Fortschritte der Zoologie, vol. 30, pp. 237-239, 1985.

[35] M. C. Baker and A. E. Baker, "Niche relationships among six species of shorebirds on their wintering and breeding ranges," Ecological Monographs, vol. 43, no. 2, pp. 193-212, 1973.

[36] M. Andersson, “On optimal predator search," Theoretical Population Biology, vol. 19, no. 1, pp. 58-86, 1981.

[37] M. W. Pienkowski, "Changes in the foraging pattern of plovers in relation to environmental factors," Animal Behaviour, vol. 31, no. 1, pp. 244-264, 1983.

[38] N. B. Metcalfe, "Prey detection by intertidally feeding Lapwing," Zeitschrift für Tierpsychologie, vol. 67, no. 1-4, pp. 45-57, 1985.

[39] StatSoft, STATISTICA (Data Analysis Software System). Version 8.0, StatSoft, Tulsa, Okla, USA, 2007, http://www.statsoft.com/.

[40] K. Kober, Foraging ecology and habitat use of wading birds and shorebirds in the mangrove ecosystem of the Caéte Bay, Northeast Pará, Brazil [Dissertation for Doctoral Degree], University of Bremen, Bremen, Germany, 2004.

[41] C. Battisti, G. Dodaro, and D. Franco, "The data reliability in ecological research: a proposal for a quick self-assessment tool," Natural History Sciences, vol. 1, no. 2, pp. 75-79, 2014.

[42] E. McCrum-Gardner, "Which is the correct statistical test to use?" British Journal of Oral and Maxillofacial Surgery, vol. 46, no. 1, pp. 38-41, 2008.

[43] J. Burger, "Abiotic factors affecting migrant shorebirds," in Shorebirds: Migration and Foraging Behavior, J. Burger and B. L. Olla, Eds., pp. 125-202, Plenum Press, New York, NY, USA, 1984.

[44] J. Burger, M. A. Howe, D. C. Hahn, and J. Chase, "Effects of tide cycles on habitat selection and habitat partitioning by migrating shorebirds," The Auk, vol. 94, no. 4, pp. 743-758, 1977.

[45] T. C. Moermond, "A functional approach to foraging: morphology, behavior and the capacity to exploit," in Studies in Avian Biology, M. L. Morrison, C. J. Ralph, J. Verner, and J. R. Jehl Jr., Eds., vol. 13, pp. 427-430, Cooper Ornithological Society, San Diego, Calif, USA, 1990.
[46] S. E. A. Le V. Dit Durell, "Individual feeding specialisation in shorebirds: population consequences and conservation implications," Biological Review, vol. 75, no. 4, pp. 503-518, 2000.

[47] G. Fernández and D. B. Lank, "Foraging behaviour of nonbreeding Western Sandpipers Calidris mauri as a function of sex, habitat and flocking," Ibis, vol. 150, no. 3, pp. 518-526, 2008.

[48] P. Esselink and L. Zwarts, "Seasonal trend in burrow depth and tidal variation in feeding activity of Nereis diversicolor," Marine Ecology Progress Series, vol. 56, pp. 243-254, 1989.

[49] C. R. Isola, M. A. Colwell, O. W. Taft, and R. J. Safran, "Interspecific differences in habitat use of shorebirds and waterfowl foraging in managed wetlands of California's San Joaquin Valley," Waterbirds, vol. 23, no. 2, pp. 196-203, 2000.

[50] L. M. Weber and S. M. Haig, "Shorebird use of South Carolina managed and natural coastal wetlands," The Journal of Wildlife Management, vol. 60, no. 1, pp. 73-82, 1996.

[51] M. A. Colwell and S. L. Landrum, "Nonrandom shorebird distribution and fine-scale variation in prey abundance," The Condor, vol. 95, no. 1, pp. 94-103, 1993.

[52] D. P. Whitfield, "Individual feeding specializations of wintering turnstone Arenaria interpres," Journal of Animal Ecology, vol. 59, no. 1, pp. 193-211, 1990.

[53] Y. Zharikov and G. A. Skilleter, "Sex-specific intertidal habitat use in subtropically wintering bar-tailed godwits," Canadian Journal of Zoology, vol. 80, no. 11, pp. 1918-1929, 2002.

[54] B. A. Harrington, "Morphometric variation and habitat use of Semipalmated Sandpipers during a migratory stopover," Journal of Field Ornithology, vol. 53, no. 3, pp. 258-262, 1982.

[55] A. Barbosa, "Foraging strategies and their influence on scanning and flocking behaviour of waders," Journal of Avian Biology, vol. 26, no. 3, pp. 182-186, 1995.

[56] M. W. Pienkowski, "Differences in habitat requirements and distribution patterns of plovers and sandpipers," Verhandlungen der Ornithologischen Gesellschaft in Bayern, vol. 23, pp. 105-124, 1981. 

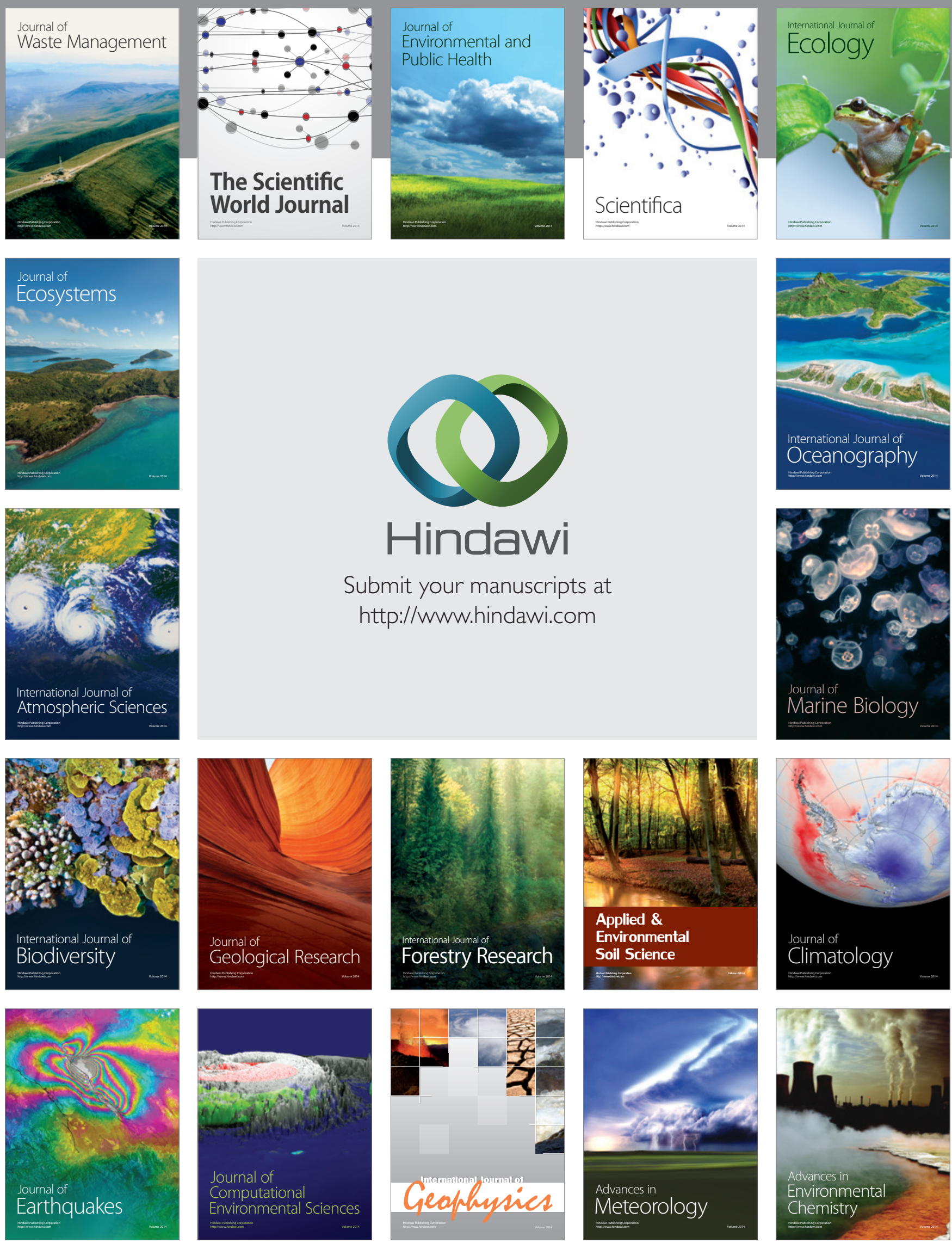